\title{
The added value of quantitative 18F-FDG-PET/CT parameters in the assessment of pulmonary lymphangitic carcinomatosis in lung cancer
}

\author{
Dexter P. Mendoza, Subba R. Digumarthy \\ Division of Thoracic Imaging and Intervention, Department of Radiology, Massachusetts General Hospital, Boston, MA, USA \\ Correspondence to: Dr. Subba R. Digumarthy. Department of Radiology, Massachusetts General Hospital, 55 Fruit Street, Founders 202, Boston, MA \\ 02114, USA. Email: sdigumarthy@mgh.harvard.edu. \\ Provenance: This is an invited article commissioned by the Section Editor Jun Zhou (Department of Nuclear Medicine, Zhongshan Hospital, Fudan \\ University, Shanghai, China). \\ Comment on: Jreige M, Dunet V, Letovanec I, et al. Pulmonary lymphangitic carcinomatosis: diagnostic performance of HRCT and 18F-FDG-PET/ \\ CT in correlation to clinical pathologic outcome. J Nucl Med 2019. [Epub ahead of print].
}

Submitted Aug 21, 2019. Accepted for publication Sep 11, 2019.

doi: $10.21037 /$ jtd.2019.10.10

View this article at: http://dx.doi.org/10.21037/jtd.2019.10.10

Lymphangitic carcinomatosis is the infiltration and spread of malignant cells through the pulmonary lymphatics. In non-lung malignancies such as breast, gastric, and colon cancer, this typically occurs via hematogenous dissemination of tumor cells to the lung with subsequent extension to the lymphatics (1-3). In primary lung cancers, on the other hand, lymphangitic carcinomatosis may also result from direct invasion from the tumor, through direct spread from pleural metastasis, or via retrograde extension from a metastatic lymph node (4-6).

The high-resolution computed tomography (HRCT) findings of lymphangitic carcinomatosis are well described. The typical features are nodular thickening of the axial and peripheral subpleural interstitium, with relative sparing of the parenchymal (i.e., intralobular) interstitium (3,7-10). The utility of $18 \mathrm{~F}$-fluorodeoxyglucose positron emission tomography (18F-FDG-PET) in detecting the presence of lymphangitic carcinomatosis has also been investigated. Our group was the first to report both qualitative and quantitative increase in the distribution of 18 -FDG in pulmonary lymphangitic carcinomatosis with increased $18 \mathrm{~F}-\mathrm{FDG}$ uptake in the affected lung compared to the healthy contralateral lung and the lungs of healthy controls (11). There was a significant increase in the ratio of the standard uptake value (SUV) of the affected lung to contralateral normal lung and a decrease in the ratio of mediastinal blood pool SUV to the affected lung. These findings were subsequently confirmed by another small series, with patterns of $18 \mathrm{~F}-\mathrm{FDG}$ uptake varying from localized, segmental, or lobar to diffuse and extensive (12). Our group also later showed that PET has high sensitivity and near $100 \%$ specificity in determining the presence of lymphangitic carcinomatosis (13). The significant drawback of these studies was lack of histopathological confirmation of lymphangitic carcinomatosis in many cases.

In the recent original research entitled, "Pulmonary lymphangitic carcinomatosis: diagnostic performance of HRCT and 18F-FDG-PET/CT in correlation to clinical pathologic outcome" published in fournal of Nuclear Medicine, Jreige and colleagues reviewed the HRCT and qualitative and quantitative 18F-FDG-PET/CT findings of 94 patients with lung cancer who had undergone surgical tumor resection without neoadjuvant therapy (14). Out of this cohort, 69 patients had histologic findings of lymphangitic carcinomatosis. The authors report that quantitative 18F-FDG-PET/CT parameters may perform better compared to either HRCT findings or qualitative 18F-FDG-PET/CT findings alone in the assessment of lymphangitic carcinomatosis (14). Specifically, the authors report that maximal peritumoral SUV $\left(\mathrm{SUV}_{\max }\right)$ greater than $2.1 \mathrm{~g} / \mathrm{mL}$ (sensitivity: 97\%; specificity: $92 \%$ ) and mean peritumoral SUV (SUV $\left.{ }_{\text {mean }}\right)$ with a cut-off of $1.2 \mathrm{~g} / \mathrm{mL}$ (sensitivity: 94\%; specificity: $88 \%$ ) had significantly higher sensitivities and specificities in detecting lymphangitic 

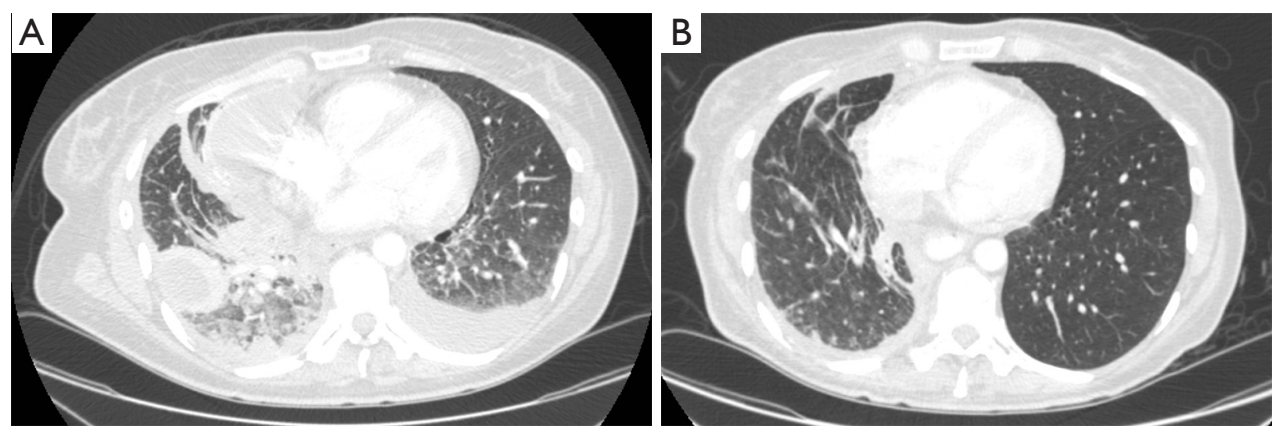

Figure 1 Fifty-four-year-old female with biopsy proven ALK-positive non-small cell lung cancer. (A) Initial chest CT shows a right lower lobe mass with associated pleural metastases and malignant pleural effusion. There is also nodular septal thickening and ground-glass opacities in the lower lobes consistent with lymphangitic carcinomatosis; (B) follow-up chest CT 2 months following initiation of ALK-targeted tyrosine kinase inhibitor shows marked treatment response with decreased size of primary lung mass and improvement of associated pleural metastases and lymphangitic carcinomatosis. ALK, anaplastic lymphoma kinase.

carcinomatosis compared to peribronchovascular thickening (sensitivity: 69\%; specificity $83 \%$ ) and compared to qualitatively increased peritumoral FDG uptake (sensitivity: $94 \%$; specificity $84 \%$ ). The strength of this study is confirmatory histopathology in all the cases, the ultimate standard of reference.

However, as with other studies that compare individual accuracy of different diagnostic tests, this paper also has some limitations. One question that the study did not address is if the quantitative parameters would still perform better compared to that of combined findings of HRCT and qualitative PET. Interpretation of PET findings is typically performed in conjunction with or following review of the HRCT, or vice versa, when available. Understandably, practice patterns vary locally and regionally; however, in our experience, it is in uncommon for PET imaging to be obtained without prior or concurrent HRCT images. In fact, all of the patients in this study had both HRCT and PET imaging as part of the initial staging. It would have been interesting if authors also explored this aspect of integrated diagnostic interpretation.

In addition, the diagnostic accuracy of HRCT was assessed using isolated imaging variables (e.g., peribronchovascular thickening, nodular lines, satellite nodules, etc.), as opposed to the gestalt HRCT imaging findings suggestive of lymphangitic carcinomatosis, potentially underestimating sensitivity and specificity of HRCT. It would be interesting to see how the combined imaging findings on HRCT and the combined results of both HRCT and qualitative PET would compare to the quantitative PET parameters. It would also be interesting to see if and to what degree the addition of quantitative metrics to routine HRCT and PET/CT would improve diagnostic accuracy in lymphangitic carcinomatosis.

Finally, the cohort consisted mostly of patients with earlier stage lung cancers, with only two patients with stage IV disease. While this is inevitable given the design of the study, which requires resection for histologic confirmation, this may limit the applicability of the findings in patients with more advanced disease.

Despite these limitations, the findings of Jreige et al. have potential in improving the accurate identification of lymphangitic carcinomatosis, which has the treatment and prognostic implications. Lymphangitic carcinomatosis is associated with worse prognosis in various extrapulmonary malignancies (15-17). The prognostic impact of lymphangitic carcinomatosis in the setting of primary lung malignancies, however, is less clear. In fact, the latest iteration of the TNM staging for lung cancer still does not include lymphangitic in the staging of primary lung malignancies due to the paucity of data (18). While it may be intuitive to conclude that lymphangitic carcinomatosis is suggestive of a more advanced malignancy and carries poorer prognosis in lung cancer, we have recently reported that non-small cell lung cancer with specific mutations [i.e., anaplastic lymphoma kinase (ALK) rearrangements] may have higher propensity for the development of lymphangitic carcinomatosis compared to other molecular subsets (Figure 1) (19,20). Targeted therapy with specific tyrosine kinase inhibitors can potentially improve outcomes in this subset of patients. These make the reliable detection of lymphangitic carcinomatosis even more critical. 
The authors' findings may also have implications in research. One of the advantages of using quantitative PET parameters is that they provide objective measures that are reliable and reproducible $(21,22)$. These parameters are of potential use in radiomics and machine learning as it pertains to diagnosis and prognostication in various malignancies and may facilitate future research efforts (23-25).

In conclusion, the authors have demonstrated that quantitative 18F-FDG-PET/CT parameters have high sensitivities and specificities in detecting lymphangitic carcinomatosis in the setting of untreated lung cancer and support these findings with pathology correlation. Further validation in larger, more inclusive cohorts is necessary to determine the reproducibility of reported quantitative parameters. Nevertheless, the results may prove helpful in future clinical and research efforts.

\section{Acknowledgments}

None.

\section{Footnote}

Conflicts of Interest: SR Digumarthy: provides independent image analysis for hospital contracted clinical research trials programs for Merck, Pfizer, Bristol Mayer Squibb, Novartis, Roche, Polaris, Cascadian, Abbvie, Gradalis, Bayer, Zai laboratories. Received honorarium from: Siemens. Mendoza DP has no conflicts of interest to declare.

Ethical Statement: The authors are accountable for all aspects of the work in ensuring that questions related to the accuracy or integrity of any part of the work are appropriately investigated and resolved.

\section{References}

1. Davis SD. CT evaluation for pulmonary metastases in patients with extrathoracic malignancy. Radiology 1991;180:1-12.

2. Biswas A, Sriram PS. Getting the whole picture: lymphangitic carcinomatosis. Am J Med 2015;128:837-40.

3. Ikezoe J, Godwin JD, Hunt KJ, et al. Pulmonary lymphangitic carcinomatosis: chronicity of radiographic findings in long-term survivors. AJR Am J Roentgenol 1995;165:49-52.

4. Janower ML, Blennerhassett JB. Lymphangitic spread of metastatic cancer to the lung. A radiologic-pathologic classification. Radiology 1971;101:267-73.

5. Masson RG, Krikorian J, Lukl P, et al. Pulmonary microvascular cytology in the diagnosis of lymphangitic carcinomatosis. N Engl J Med 1989;321:71-6.

6. Soares FA, Pinto AP, Landell GA, et al. Pulmonary tumor embolism to arterial vessels and carcinomatous lymphangitis. A comparative clinicopathological study. Arch Pathol Lab Med 1993;117:827-31.

7. Honda $\mathrm{O}$, Johkoh T, Ichikado K, et al. Comparison of high resolution CT findings of sarcoidosis, lymphoma, and lymphangitic carcinoma: is there any difference of involved interstitium? J Comput Assist Tomogr 1999;23:374-9.

8. Johkoh T, Ikezoe J, Tomiyama N, et al. CT findings in lymphangitic carcinomatosis of the lung: correlation with histologic findings and pulmonary function tests. AJR Am J Roentgenol 1992;158:1217-22.

9. Pasławski M, Krzyzanowski K, Złomaniec J. Lymphangitis carcinomatosa in thin section computed tomography. Ann Univ Mariae Curie Sklodowska Med 2004;59:1-5.

10. Munk PL, Müller NL, Miller RR, et al. Pulmonary lymphangitic carcinomatosis: CT and pathologic findings. Radiology 1988;166:705-9.

11. Digumarthy SR, Fischman AJ, Kwek BH, et al. Fluorodeoxyglucose positron emission tomography pattern of pulmonary lymphangitic carcinomatosis. J Comput Assist Tomogr 2005;29:346-9.

12. Acikgoz G, Kim SM, Houseni M, et al. Pulmonary lymphangitic carcinomatosis (PLC): spectrum of FDGPET findings. Clin Nucl Med 2006;31:673-8.

13. Prakash P, Kalra MK, Sharma A, et al. FDG PET/CT in assessment of pulmonary lymphangitic carcinomatosis. AJR Am J Roentgenol 2010;194:231-6.

14. Jreige M, Dunet V, Letovanec I, et al. Pulmonary lymphangitic carcinomatosis: diagnostic performance of HRCT and 18F-FDG-PET/CT in correlation to clinical pathologic outcome. J Nucl Med 2019. [Epub ahead of print].

15. Klimek M. Pulmonary lymphangitis carcinomatosis: systematic review and meta-analysis of case reports, 19702018. Postgrad Med 2019;131:309-18.

16. Moubax K, Wuyts W, Vandecaveye V, et al. Pulmonary lymphangitic carcinomatosis as a primary manifestation of gastric carcinoma in a young adult: a case report and review of the literature. BMC Res Notes 2012;5:638.

17. Charest M, Armanious S. Prognostic implication of the lymphangitic carcinomatosis pattern on perfusion lung scan. Can Assoc Radiol J 2012;63:294-303.

18. Rami-Porta R, Bolejack V. Reply to "Inclusion of 
Lymphangitis as a Descriptor in the New TNM Staging of Lung Cancer: Filling Up the Blank Spaces.” J Thorac Oncol 2015;10:e119-20.

19. Mendoza DP, Stowell J, Muzikansky A, et al. Computed Tomography Imaging Characteristics of Non-Small-

Cell Lung Cancer With Anaplastic Lymphoma Kinase Rearrangements: A Systematic Review and Meta-Analysis. Clin Lung Cancer 2019;20:339-49.

20. Mendoza DP, Dagogo-Jack I, Chen T, et al. Imaging characteristics of BRAF-mutant non-small cell lung cancer by functional class. Lung Cancer 2019;129:80-4.

21. de Langen AJ, Vincent A, Velasquez LM, et al. Repeatability of 18F-FDG uptake measurements in

Cite this article as: Mendoza DP, Digumarthy SR. The added value of quantitative $18 \mathrm{~F}$-FDG-PET/CT parameters in the assessment of pulmonary lymphangitic carcinomatosis in lung cancer. J Thorac Dis 2019;11(11):E239-E242. doi: 10.21037/ jtd.2019.10.10 tumors: a metaanalysis. J Nucl Med 2012;53:701-8.

22. Lodge MA. Repeatability of SUV in Oncologic $18 \mathrm{~F}-\mathrm{FDG}$ PET. J Nucl Med 2017;58:523-32.

23. Digumarthy SR, Padole AM, Gullo RL, et al. Can CT radiomic analysis in NSCLC predict histology and EGFR mutation status? Medicine (Baltimore) 2019;98:e13963.

24. Mak RH, Digumarthy SR, Muzikansky A, et al. Role of $18 \mathrm{~F}$-fluorodeoxyglucose positron emission tomography in predicting epidermal growth factor receptor mutations in non-small cell lung cancer. Oncologist 2011;16:319-26.

25. Cook GJ, Azad G, Owczarczyk K, et al. Challenges and Promises of PET Radiomics. Int J Radiat Oncol Biol Phys 2018;102:1083-9. 